\title{
Radioprotection pendant la première visite générale de la centrale nucléaire des Ardennes $\left(^{*}\right)$
}

\author{
J.-J. MARTIN (**), R. RIEHL (***)
}

(Manuscrit reçu le 17 septembre 1975)

\begin{abstract}
RÉSUMÉ
L'arrêt pour rechargement en 1974 de la centrale des Ardennes a comporté des travaux exceptionnels impliquant l'intervention d'un effectif important. Profitant de l'expérience acquise lors des arrêts précédents, des améliorations ont été apportées aux moyens de radioprotection du personnel.

Le bilan des doses collectives a permis d'estimer le coût dosimétrique de certains travaux spécifiques en vue de calculs prévisionnels pour les installations nouvelles.
\end{abstract}

\begin{abstract}
The 1974 refueling outage included exceptional works implying large manpower. Experience gained during previous outages was put into practice to improve radiation procedures.

Evaluation of collective doses allowed to estimate the dosimetric cost of a number of specific maintenance works with a view to provisional calculation for new installations.
\end{abstract}

(*) Communication présentée à la Conférence nucléaire européenne organisée à Paris du 21 au 25 avril 1975, par la Société européenne de l'Énergie nucléaire et l'American nuclear Society (First published in the Proceedings of the European nuclear conference, in Progress in nuclear energy series, Pergamon press, Oxford, New York, Paris, 1976).

(**) Électricité de France, Service de la Production thermique, Département de Radioprotection, B.P. $\mathrm{n}^{\circ} 120,93203$ Saint-Denis.

${ }^{(* * *)}$ Centrale nucléaire des Ardennes, S.E.N.A., Chooz, France.

RADIOPROTECTION, VOL, $11-\mathrm{N}^{\circ} 1$ 


\section{INTRODUCTION}

L'arrêt pour rechargement, en 1974, de la centrale nucléaire des Ardennes, premier réacteur à eau pressurisée en service en France depuis 1967, est le premier arrêt pendant lequel l'ensemble du circuit primaire (cuve du réacteur, tuyauteries primaires principales et générateurs de vapeur) a été inspecté. De plus, un travail très important a été entrepris au voisinage immédiat des tuyauteries primaires en vue de réduire leur possibilité de mouvement dans l'hypothèse d'une rupture.

Comme les arrêts précédents avaient entraîné des doses individuelles et collectives assez élevées, particulièrement durant l'arrêt de l'année 1973 en raison d'une contamination importante du circuit primaire, on pouvait craindre des doses plus grandes dues à ces travaux supplémentaires.

On a profité de l'expérience acquise pendant les années précédentes dans le domaine de la radioprotection en vue d'obtenir les doses les plus faibles possibles, compte tenu de l'importance des travaux au cours de cet arrêt particulier. Les efforts exercés dans ce but sont pleins d'enseignement pour Électricité de France à une époque où un grand nombre de réacteurs à eau légère sont mis en chantier en France.

Nous allons décrire l'organisation en matériel et en personnel mise en place, puis quelques travaux particuliers impliquant des problèmes spécifiques de radioprotection; enfin, nous donnerons le bilan des expositions observées chez les travailleurs.

\section{ORGANISATION}

L'organisation des travaux lors de l'arrêt de tranche 1974 a apporté des améliorations aux postes de contrôle de la contamination externe, aux moyens d'estimation des doses externes et des contaminations internes, à l'accès à la piscine du réacteur et aux moyens de décontamination des zones de travail.

Les contrôles de contamination externe des travailleurs étaient effectués successivement en trois emplacements. Le premier a été mis en place à la sortie de la caverne du réacteur, en vue d'éviter une dispersion de la contamination dans un long couloir qui s'étend de la caverne du réacteur à l'accès principal. La protection du détecteur permettait, en ce point, une discrimination satisfaisante entre les vêtements contaminés et peu contaminés, mais n'était pas adaptée à un contrôle de contamination cutanée en raison du bruit de fond élevé. Il faut noter que les vêtements spécialisés (survêtements) utilisés sur les lieux de travail contaminés étaient systématiquement retirés à la sortie de la zone de travail. Le deuxième contrôle, situé à l'accès principal, per- 
mettait de décider si les vêtements pouvaient être à nouveau utilisés ou devaient être décontaminés. Enfin, après déshabillage, la contamination cutanée était contrôlée avec un compteur à grande surface et fenêtre mince dans un bruit de fond très faible. Les agents présentant des traces de contamination étaient dirigés sur l'infirmerie.

A chacun de ces postes de contrôle, un gardien s'assurait que les travailleurs s'habillaient ou se contrôlaient correctement et fournissait le linge propre ou envoyait le linge contaminé à la laverie. Le surveillant de l'accès principal vérifiait également que les travailleurs utilisaient les dosimètres conformément aux consignes.

L'irradiation externe des agents est obligatoirement suivie en France par des dosimètres photographiques sous la responsabilité de l'employeur. Pour connaître la dose absorbée plus rapidement, on utilise des dosimètres électroscopes à lecture directe, mais la précision dans le cumul et la lecture de ces dosimètres n'est pas suffisante lorsque la dose approche la dose réglementaire maximale admissible. On a observé aussi que les doses données par ces dosimètres sont souvent un peu plus faibles (environ $20 \mathrm{p}$. cent) que les doses données par les films. En conséquence, on a doté chaque travailleur d'un dosimètre thermoluminescent, lu périodiquement ou à l'occasion de travaux particuliers.

Un dispositif d'anthropogammamétrie simplifiée a été mis en place en vue de détecter, chez les agents susceptibles d'être contaminés, une contamination interne qui aurait nécessité des mesures plus élaborées dans un service spécialisé. Ce dispositif, exploité par l'infirmier, effectuait une spectrométrie au niveau du thorax de l'individu assis et permettait un seuil de détection nettement inférieur à $1 \mathrm{p}$. cent de la quantité maximale admissible dans l'organisme entier.

Afin de réduire le temps de présence des agents travaillant dans la piscine du réacteur après qu'elle eut été vidée, un escalier a été mis en place. De fait, on avait observé pendant les années précédentes que les agents restaient au fond de la piscine entre deux phases de travail parce qu'il était fatigant de remonter à chaque fois l'échelle de $9 \mathrm{~m}$, ou avaient des difficultés à remonter des charges (fûts de déchets par exemple). L'escalier a ainsi réduit les doses inutiles et facilité l'enlèvement du petit matériel radioactif. De plus, la machine de chargement a été équipée d'une potence avec un treuil pour faciliter la remontée des petites charges.

Enfin, des contrôles de contamination des zones de circulation ont été faits périodiquement en vue de déclencher des opérations de décontamination avant que ces zones ne constituent une source de contamination pour les zones avoisinantes.

L'effectif affecté à des fonctions liées à la radioprotection pendant l'arrêt de tranche a atteint de l'ordre de 35 agents dont 9 techniciens de radioprotection en roulement et 19 agents d'entreprises extérieures affectés au gardiennage des sas, à la laverie, à la décontamination des sols et à l'enfûtage des déchets.

voL. $11-\mathrm{N}^{0} 1$ 


\section{RÉSULTATS}

Les travaux de l'arrêt de tranche 1974 se sont déroulés dans des conditions de contamination et de débit de dose plus faibles qu'en 1973, ainsi qu'il apparaît sur le tableau $\mathbf{I}$.

\section{TABLEAU I}

RÉSUltats COMPARÉS DES ANNÉES 1973 ET 1974

\begin{tabular}{|c|c|c|c|c|}
\hline & & Unité & 1973 & 1974 \\
\hline \multicolumn{2}{|l|}{ Activité $\beta$ globale } & & & \\
\hline \multicolumn{2}{|c|}{ - de l'eau primaire avant arrêt } & $10^{-3} \mathrm{Ci} / \mathrm{m}^{3}$ & 19000 & 1500 \\
\hline \multicolumn{2}{|c|}{ - de l'eau de la piscine réacteur } & $»$ & 28 & 2,2 \\
\hline \multicolumn{5}{|l|}{ Activité en tritium } \\
\hline \multicolumn{2}{|c|}{ - de l'eau primaire avant arrêt } & $»$ & 3100 & 2860 \\
\hline \multicolumn{2}{|c|}{ - de l'eau de la piscine réacteur } & » & 140 & 130 \\
\hline \multicolumn{5}{|l|}{ Activité $\beta$ globale } \\
\hline \multicolumn{2}{|c|}{ - de l'air de la caverne réacteur $\mathrm{J}+1$ (1) } & $10^{-12} \mathrm{Ci} / \mathrm{m}^{3}$ & 370 & 100 \\
\hline & $\mathrm{J}+15$ & $»$ & 100 & 30 \\
\hline \multirow{2}{*}{ Activité en ${ }^{131}$ I } & $\mathbf{J}+1$ & " & 40 & 3 \\
\hline & $\mathrm{J}+15$ & $»$ & $<1$ & $<1$ \\
\hline \multicolumn{5}{|l|}{ Débit de dose à l'extérieur } \\
\hline - d'une pompe primaire & $\mathrm{J}+11$ & $\mathrm{mrem} / \mathrm{h}$ & 700 & 50 \\
\hline - d'une vanne primaire & $\mathrm{J}+11$ & " & 450 & 100 à 200 \\
\hline - d'un fond de générateur & $\mathrm{J}+11$ & » & 500 & 100 \\
\hline
\end{tabular}

(1) $\mathrm{J}$ : jour de l'arrêt du réacteur.

Malgré la durée de l'arrêt, la dose collective a été sensiblement équivalente pour le personnel de la SENA en 1974 et en 1973 (175 hommes-rems contre 167 hommes-rems d'après les films dosimètres). En raison de l'importance des travaux, la dose du personnel prenant une part active aux opérations a été suivie par des dosimètres thermoluminescents, s'ajoutant aux films dosimètres réglementaires. Pour 615 agents des entreprises contrôlés de cette façon, la dose collective atteint 727 hommes-rems. Pour 89 des agents SENA les plus exposés, elle atteint 160 hommes-rems. La dose moyenne individuelle ressort ainsi à 1,18 rem pour le personnel d'entreprise et 1,8 rem pour les agents SENA. Il faut souligner le caractère exceptionnel des travaux engagés. 
Des travaux particuliers ont été suivis avec une attention soutenue. Ainsi, l'enlèvement et la remise en place du calorifuge des boucles primaires pour le contrôle des soudures ou la mise en place des structures anti-fouettement autour des tuyauteries primaires ont constitué un long travail dans un champ de rayonnement de 30 à $200 \mathrm{mrem} / \mathrm{h}$ avec quelques risques de contamination.

\section{TABLEAU II}

DOSES OBSERVÉES

$\begin{array}{lrccc}\text { Dose collective agents SENA (films dosimètres) } & \text { h.rem } & 175 & \text { (167)(1) } \\ \text { Dose collective agents SENA (thermoluminescence) } & \text { h.rem } & 160 & \\ \text { Dose moyenne agents SENA (thermoluminescence) } & \text { rem } & 1,8 & \\ \text { Dose collective agents d'entreprise (thermoluminescence) } & \text { h.rem } & 727 & \\ \text { Dose moyenne agents d'entreprise (thermoluminescence) } & \text { rem } & 1,18 & \\ \text { Nettoyage de la piscine } & \text { h.rem } & 9,7 & \text { (27) } \\ \text { Décalorifugeage des tuyauteries primaires } & \text { h.rem } & 5,2 & \\ & \text { h.rem/m } & 0,09 & \\ \text { Décalorifugeage des boítes à eau GV } & \text { h.rem } & 3,6 & \\ \text { Recalorifugeage des boítes à eau } & \text { h.rem } & 18 & \\ \text { Ouverture et fermeture du trou d'homme du pressuriseur } & \text { h.rem } & 4,5 & \\ \text { Désaccouplement des barres de commande } & \text { h.rem } & 2,4 & (6,5) \\ \text { Contrôle de soudure des tuyauteries primaires par ressuage } & \text { h.rem } / \mathrm{m} & 0,043 & \\ \text { Contrôle de la bride de cuve ultra-sons } & \text { h.rem } / \mathrm{m}^{2} & 0,17 & \\ \text { Contrôle des soudures de tuyauteries } & \text { h.rem } / \mathrm{m} & 0,13 & \end{array}$

(1) Les valeurs entre parenthèses correspondent aux résultats de 1973.

Le piquage du béton nécessaire pour la mise en place des structures a conduit à travailler dans une atmosphère empoussiérée; heureusement, seuls les premiers millimètres de béton présentaient une contamination.

Les contrôles du pressuriseur après décalorifugeage et ouverture du trou d'homme ont posé des difficultés en raison du débit de dose élevé (point à $400 \mathrm{mrem} / \mathrm{h}$ ) et d'une contamination importante résultant d'une fuite d'eau primaire. Deux générateurs de vapeur ont subi des examens des faisceaux de tubes dans une ambiance de 5 à $12 \mathrm{rem} / \mathrm{h}$ limitant la durée des interventions.

Les principaux résultats apparaissent dans le tableau II.

La contamination en tritium de l'eau de la piscine réacteur a entraîné une contamination de l'atmosphère de l'enceinte du réacteur comprise entre 2 et $5 \cdot 10^{-7} \mathrm{Ci} / \mathrm{m}^{3}$, soit 0,1 à $0,25 \mathrm{CMA}$, la ventilation étant en service $\left(100000 \mathrm{~m}^{3} / \mathrm{h}\right)$. Cette contamination correspond à la charge en eau ajoutée par l'eau de la piscine à la charge en eau de l'air extérieur. Elle diminue lorsque l'on s'éloigne de la piscine.

Le nettoyage de la piscine après les opérations de déchargement du combustible a permis de réduire d'un facteur 10 en moyenne le débit de 
dose au fond de la piscine avant les opérations de fermeture du couvercle de cuve. On a constaté que les produits d'activation tels que cobalt et manganèse, ainsi que les émetteurs $\alpha$, étaient plus facilement enlevés que les produits de fission, tels que césium et cérium.

\section{CONCLUSIONS}

Les efforts faits dans l'organisation des travaux ont certainement permis de réduire l'exposition du personnel. Avec des tâches accrues et un arrêt de presque cinq mois au lieu de cinq semaines l'année précédente, la dose collective du personnel SENA est restée sensiblement la même en 1974 et en 1973. Les doses collectives prises par les personnels d'entreprise et de SENA traduisent l'ampleur exceptionnelle des travaux entrepris.

La contamination interne est restée inférieure au centième de la quantité maximale admissible dans l'organisme entier pour les quelques agents susceptibles d'être légèrement contaminés. Il semble donc que les moyens de protection contre la contamination ont été suffisamment efficaces.

De tels résultats n'ont pu être obtenus que moyennant l'affectation d'un personnel supplémentaire important aux tâches de radioprotection, soit pour les nombreuses tâches d'intendance, soit pour l'encadrement du personnel d'entreprises. Cette nécessité se fait d'autant plus sentir que ce personnel d'entreprises n'est malheureusement pas encore au fait des conditions particulières qu'impose le travail en milieu radioactif. Il faut souhaiter qu'une information suffisante pourra être fournie en temps utile par la personne compétente dépendant de chaque employeur pour tout le personnel qui devra intervenir sur les centrales en cours de mise en service en France. 